Reihe Rechtswissenschaft

Band 176 


\title{
Rechtsfolgen der Manipulation bei juristischen Prüfungen
}

\author{
Bettina Barten
}

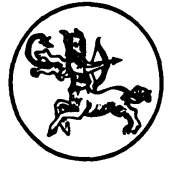

Centaurus Verlag \& Media UG 1994 
Die Deutsche Bibliothek - CIP-Einheitsaufnahme

\section{Barten, Bettina:}

Rechtsfolgen der Manipulation

bei juristischen Prüfungen / Bettina Barten. -

Pfaffenweiler : Centaurus-Verl.-Ges., 1994

(Reihe Rechtswissenschaft ; Bd. 176)

Zugl.: Trier, Univ., Diss., 1994

ISBN 978-3-89085-966-8 ISBN 978-3-86226-455-1 (eBook)

DOI $10.1007 / 978-3-86226-455-1$

\section{NE: GT}

\section{ISSN 0177-2805}

Alle Rechte, insbesondere das Recht der Vervielfältigung und Verbreitung sowie der Übersetzung, vorbehalten. Kein Teil des Werkes darf in irgendeiner Form (durch Fotokopie, Mikrofilm oder ein anderes Verfahren) ohne schriftliche Genehmigung des Verlages reproduziert oder unter Verwendung elektronischer Systeme verarbeitet, vervielfältigt oder verbreitet werden.

(C) CENTAURUS-Verlagsgesellschaft mit beschränkter Haftung, Pfaffenweiler 1994

Satz: Vorlage der Autorin 
Meinen Eltern 


\section{Vorwort}

Die vorliegende Arbeit wurde im Wintersemester 1993/94 vom Fachbereich Rechtswissenschaft der Universität Trier als Dissertation angenommen.

Die Problematik der Manipulation bei juristischen Prüfungen dürfte wohl jedem bekannt sein, der mit diesem Themenbereich befaßt ist. Anstoß für die vorliegende Dissertation waren einige an der Universität Trier bekanntgewordene Manipulationsfälle im Rahmen von juristischen Prüfungen; Studenten fertigten für Prüfungsteilnehmer Aufsichtsarbeiten unter deren Namen an.

Im Verlauf der Untersuchung wurde das Deutsche Richtergesetz durch das Gesetz zur Verkürzung der Juristenausbildung vom 20.11.1992 (BGBl. I, S. 1926) geändert. Hierdurch wurden unter anderem die sogenannten Leistungskontrollen als Zulassungsvoraussetzung für die Fortgeschrittenenübungen und das erste juristische Staatsexamen formell abgeschafft. Sie finden jedoch vorliegend weiterhin Berücksichtigung, da bei Abschluß der Arbeit noch keine vollständige Umsetzung der Änderungen durch die Länder und Universitäten erfolgt war. Darüber hinaus bleiben die Leistungskontrollen sowohl für die Rechtsfolgen zukünftig aufgedeckter Manipulation als auch unter dem Gesichtspunkt der Heilungsproblematik relevant.

Nach Auffassung der rheinland-pfälzischen Landesregierung erforderte die Transparenz der Ausbildungsregelungen eine Neufassung des Juristenausbildungsgesetzes und der Juristischen Ausbildungs- und Prüfungsordnung. Zum Zeitpunkt der Dissertationseinreichung existierten jedoch lediglich Entwürfe dieser Normierungen. Deshalb werden als Anhang die erst nach Fertigstellung und Einreichung der Arbeit statuierten Änderungen in einem Überblick dargestellt.

An dieser Stelle möchte ich es nicht versäumen, mich bei all jenen, die mich bei meinem Vorhaben unterstützt haben, aufrichtig zu bedanken. Aufgrund der bestehenden Vielfalt an Personen, denen ich zu Dank verpflichtet bin, möchte ich ohne Darlegung ihrer jeweiligen Verdienste insbesondere danken: Prof. Dr. Rainer Zaczyk, Dr. Jürgen Kemper, meinen Eltern, meinem Bruder, meiner Großtante Änne Lamberts, Beate Hüllen, Ref. iur. Barbara Horsmann, Cand. iur. Stefan Jaeger und insbesondere meinem Doktorvater Prof. Dr. Hans-Heiner Kühne. 


\section{$\underline{\underline{\text { Inhaltsverzeichnis }}}$}

$\begin{array}{ll}\text { A. Einleitung } & 1\end{array}$

B. Problembeschreibung 2

I. Mitteilungen der Landesjustizprüfungsämter 3

II. Mitteilungen der Dekanate juristischer Fakultäten 6

III. Akademisches ghost-writing 9

2. TEIL: BEGRIFFSBESTIMMUNG UND VERFASSUNGSRECHTLICHE GRUNDLAGEN

A. Darlegung des Untersuchungsgegenstands $\quad 11$

I. Beschreibung der untersuchten Prüfungsarten 11

1. Die Leistungskontrollen 11

2. Die großen Scheine 13

3. Das erste juristische Staatsexamen 13

4. Das zweite juristische Staatsexamen 14

5. Die Promotion 15

II. Beschreibung der die Prüfungsmanipulation vornehmenden Personen

III. Beschreibung der untersuchten Manipulationsarten 17

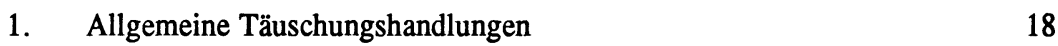

2. Vorheriges Besorgen der Prüfungsaufgabe bzw. der Lösungs-

3. Erbringung der Prüfungsleistung durch einen Dritten 20

4. Die Beeinflussung von Prüfern 20

5. Die Beeinflussung durch Prüfer 21 


\section{$\mathrm{X}$ Inhaltsverzeichnis}

IV. Abgrenzung der verbotenen Prüfungsmanipulation zum erlaubten Vorfeld

1. Abhängigkeit der zugelassenen Hilfsmittel von der Prüfungsform 21

2. Abhängigkeit der erlaubten Hilfsmittel vom technischen Fortschritt

3. Abgrenzung bei Beteiligung Dritter auf der Seite des Prüflings

4. Abgrenzung der manipulierten von der wohlwollenden bzw. strengen Korrektur

5. Abgrenzung der manipulierten von der wohlwollenden bzw. strengen Gesamtbenotung

B. Gründe, die zur Manipulation bei juristischen Prüfungen führen

C. Erforderlichkeit von unmanipulierten Prüfungen

I. Geschichtlicher Überblick

II. Allgemeine Funktionen der Prüfung

III. Spezielle Ziele der juristischen Prüfungen

IV. Erforderlichkeit der Verhinderung von Prüfungsmanipulation

1. Umgehung der Prüfungsziele

2. Verfassungsrechtliche Bestimmungen

a. Art. 12 I GG

b. Art. 33 II GG

c. Art. 3 I GG

46

d. Rechtsstaatsprinzip 
I. Der Zeitpunkt vor 1939

62

1. Der Entzug des Grades durch die verleihende Hochschule 62

2. Verlust des Grades durch Strafurteil 65

II. Der Zeitpunkt nach 1939

1. Der Entzug des Grades durch die verleihende Hochschule 65

2. Verlust des Grades durch Strafurteil 66

I. Manipulation im hochschulinternen Bereich 67

1. Die Leistungskontrollen 67

$\begin{array}{ll}\text { a. Allgemeine Täuschungshandlungen } & 67\end{array}$

aa. Strafrechtliche Beurteilung $\quad 68$

(1) $\S 263$ I StGB $\quad 68$

(2) §267 I 1. Alt. StGB 70

(3) § 271 I StGB $\quad 71$

(4) § 123 I StGB $\quad 76$

bb. Hochschulrechtliche Beurteilung $\quad 77$

(1) Leistungskontrollordnung $\quad 77$

(2) Hochschulgesetz $\quad 81$

b. Vorheriges Besorgen der Prüfungsaufgabe bzw. der $\begin{array}{ll}\text { Lösungsskizze } & 84\end{array}$

aa. Strafrechtliche Beurteilung $\quad 85$

(1) § 263 I StGB $\quad 85$

(2) § 267 I 1. Alt. StGB $\quad 85$

(3) § 271 I StGB $\quad 85$

bb. Hochschulrechtliche Beurteilung $\quad 86$

c. Anfertigung der Prüfungsleistung durch einen Dritten 89

aa. Unterzeichnung und Ablieferung der Klausur durch $\begin{array}{ll}\text { den eigentlichen Prüfling } & 89\end{array}$

(1) Strafrechtliche Beurteilung $\quad 89$

(a) § 263 I StGB $\quad 89$

(b) $\S 267$ I 1. Alt. StGB 90

(c) §271 I StGB 92 
(2) Hochschulrechtliche Beurteilung

bb. Anfertigung, Unterzeichnung und Ablieferung der Klausur durch den Dritten

(1) Strafrechtliche Beurteilung $\quad 94$

(a) § 263 I / § 267 I 1. und 3. Alt. / § 123 I StGB

(b) § 271 I StGB

(2) Hochschulrechtliche Beurteilung 96

d. Beeinflussung von Prüfern durch die Gewährung materieller oder immaterieller Vorteile $\quad 97$

aa. Strafrechtliche Beurteilung $\quad 97$

(1) § 271 I StGB 97

(2) § 333 I StGB 98

(3) § 334 I i.V.m. III Nr. 2 StGB 100

bb. Hochschulrechtliche Beurteilung 102

2. Die Übungen für Fortgeschrittene 104

a. Strafrechtliche Beurteilung 106

b. Hochschulrechtliche Beurteilung 106

3. Die Promotion 107

a. Manipulation im Rahmen der Dissertation 109

aa. Allgemeine Täuschungshandlungen 109

(1) Strafrechtliche Beurteilung 109

(a) § 263 I StGB $\quad 109$

(b) $\S 267$ I 1. Alt. StGB 110

(c) § 271 I StGB

(d) $\S 156 \mathrm{StGB}$

(2) Hochschulrechtliche Beurteilung 114

(a) Versagung vor Verleihung des Doktorgrades $\quad 114$

(b) Entziehung nach Verleihung des Doktorgrades 116

bb. Anfertigung der Dissertation durch einen Dritten 118

(1) Strafrechtliche Beurteilung $\quad 119$

(a) § 263 I StGB 119

(b) $\S 267$ I 1. Alt. StGB 119

(c) § 271 I StGB 120

(2) Hochschulrechtliche Beurteilung 123

(a) Versagung vor Verleihung des Doktorgrades 123

(b) Entziehung nach Verleihung des Doktorgrades 123

cc. Beeinflussung von Prüfern durch die Gewährung materieller oder immaterieller Vorteile $\quad 125$

(1) Strafrechtliche Beurteilung 126

(a) § 271 I StGB 126 
(b) $\$ 333$ I StGB $\quad 128$

(c) § 334 I i.V.m. III Nr. 2 StGB 128

(2) Hochschulrechtliche Beurteilung 129

(a) Versagung vor Verleihung des Doktorgrades 129

(b) Entziehung nach Verleihung des Doktorgrades 134

b. Manipulation im Rahmen des Rigorosums 136

aa. Allgemeine Täuschungshandlungen und vorheriges Besorgen der Prüfungsaufgabe bzw. der Lösungsskizze

(1) Strafrechtliche Beurteilung 136

(2) Hochschulrechtliche Beurteilung 137

(a) Versagung vor Verleihung des Doktorgrades 137

(b) Entziehung nach Verleihung des Doktorgrades 137

bb. Ablegung des Rigorosums durch einen Dritten 138

cc. Beeinflussung von Prüfern durch die Gewährung materieller oder immaterieller Vorteile

(1) Strafrechtliche Beurteilung 138

(a) § 271 I StGB 138

(b) § 333 I StGB 139

(c) §334 I i.V.m. III Nr. 2 StGB 139

(2) Hochschulrechtliche Beurteilung 139

II. Manipulation bei den juristischen Staatsexamen 139

1. Das erste Staatsexamen 140

a. Manipulation bei der schriftlichen Prüfung 140

aa. Allgemeine Täuschungshandlungen und vorheriges Besorgen der Prüfungsaufgabe bzw. der Lösungsskizze

(1) Strafrechtliche Beurteilung 141

(a) \& 263 I StGB 141

(b) § 267 I 1. Alt. StGB 142

(c) § 271 I StGB

(d) \& 123 I StGB 145

(2) Außerstrafrechtliche Beurteilung 145

bb. Anfertigung der Prüfungsleistung durch einen Dritten

(1) Unterzeichnung und Ablieferung der Klausur durch den eigentlichen Prüfling

(a) Strafrechtliche Beurteilung 149

(aa) § 263 I StGB 
(bb) § 267 I 1. Alt. StGB 150

(cc) § 271 I StGB 151

(b) Außerstrafrechtliche Beurteilung 151

(2) Anfertigung, Unterzeichnung und Ablieferung der Klausur durch den Dritten $\quad 152$

(a) Strafrechtliche Beurteilung $\quad 152$

(aa) § 263 I / § 267 I 1. und 3. Alt. / § 123 I StGB 152

(bb) § 271 I StGB 152

(b) Außerstrafrechtliche Beurteilung 153

cc. Beeinflussung von Prüfern durch die Gewährung materieller oder immaterieller Vorteile 154

(1) Strafrechtliche Beurteilung $\quad 155$

(a) § 271 I StGB $\quad 155$

(b) §333 I StGB $\quad 155$

(c) § 334 I i.V.m. III Nr. 2 StGB 156

(2) Außerstrafrechtliche Beurteilung 157

dd. Exkurs 165

(1) Versagung der Einstellung in den juristischen Vorbereitungsdienst

(2) Nichtigkeit der Ernennung $\quad 171$

(3) Rücknahme der Ernennung 171

(4) Entlassung aus dem Beamtenverhältnis 178

b. Manipulation bei der mündlichen Prüfung $\quad 180$

aa. Allgemeine Täuschungshandlungen und vorheriges Besorgen der Prüfungsaufgabe bzw. der Lösungsskizze

(1) Strafrechtliche Beurteilung 181

(2) Außerstrafrechtliche Beurteilung 182

bb. Erbringung der mündlichen Prüfungsleistung durch einen Dritten

(1) Strafrechtliche Beurteilung 182

(2) Außerstrafrechtliche Beurteilung 183

cc. Beeinflussung von Prüfern durch die Gewährung materieller oder immaterieller Vorteile

(1) Strafrechtliche Beurteilung $\quad 184$

(2) Außerstrafrechtliche Beurteilung $\quad 185$

2. Das zweite Staatsexamen 185

a. Vornahme einer "nicht schweren" Prüfungsmanipulation

aa. Dienstordnungsmaßnahmen 
bb. Entlassung aus dem Beamtenverhältnis

192

b. Vornahme einer schweren Prüfungsmanipulation

193

aa. Dienstordnungsmaßnahmen

194

bb. Entlassung aus dem Beamtenverhältnis

194

c. Vornahme einer schweren Prüfungsmanipulation mit Verwirklichung eines Straftatbestandes

195

aa. Dienstordnungsmaßnahmen

196

bb. Entlassung aus dem Beamtenverhältnis

199

B. Manipulation durch den Prüfer

202

I. Manipulation im hochschulinternen Bereich

204

1. Die Leistungskontrollen und Fortgeschrittenenübungen

a. Vorherige Herausgabe der Prüfungsaufgabe und/oder der

Lösungsskizze

204

aa. Strafrechtliche Beurteilung

204

(1) § 203 II Nr. 1 StGB

204

(2) $\S 331$ I StGB

205

(3) § 332 I i.V.m III Nr. 1 StGB

210

(4) § 353 b I Nr. 1 StGB

212

bb. Außerstrafrechtliche Beurteilung

213

(1) Dienstordnungsmaßnahmen

213

(2) Sonstige Maßnahmen

216

b. Manipulation im Rahmen der Korrektur und/oder der Gesamtbenotung der Prüfungsleistung

aa. Strafrechtliche Beurteilung

(1) § 332 I i.V.m. III Nr. 2 StGB

(2) § 348 I StGB

bb. Außerstrafrechtliche Beurteilung

2. Die Promotion

a. Vorherige Herausgabe der Prüfungsaufgabe und/oder der

Lösungsskizze

aa. Strafrechtliche Beurteilung

bb. Außerstrafrechtliche Beurteilung

b. Manipulation im Rahmen der Korrektur und/oder der Gesamtbenotung der Prüfungsleistung

aa. Strafrechtliche Beurteilung

(1) § 263 I StGB

(2) § 271 I StGB

(3) § 331 I, § 332 I i.V.m. III Nr. 2 StGB 
II. Manipulation bei den juristischen Staatsexamen

1. Vorherige Herausgabe der Prüfungsaufgabe und/oder der Lösungsskizze

a. Strafrechtliche Beurteilung

b. Außerstrafrechtliche Beurteilung

2. Manipulation im Rahmen der Korrektur und/oder der Gesamtbenotung der Prüfungsleistung

b. Außerstrafrechtliche Beurteilung

III. Folgen der Manipulation für die getroffene Prüfungsentscheidung

C. Strafbarkeit des Prüflings im Falle der durch den Prüfer vorgenommenen Manipulation

D. Manipulation durch Dritte

I. Manipulation im hochschulinternen Bereich 243

1. Unerlaubte Hilfeleistung durch den Dritten 243

a. Strafrechtliche Beurteilung 244

aa. § 263 I / § 271 I StGB

bb. \& 267 I 1. Alt. StGB 244

b. Außerstrafrechtliche Beurteilung 245

2. Eintritt in die Position des eigentlichen Prüfungskandidaten 247

a. Strafrechtliche Beurteilung 247

aa. § 263 I / § 271 I StGB 247

bb. $\S 267$ I 1. und 3. Alt. StGB 248

cc. $\S 123$ I StGB 250

b. Außerstrafrechtliche Beurteilung 252

II. Manipulation bei den juristischen Staatsexamen 252

1. Unerlaubte Hilfeleistung durch den Dritten 252

a. Strafrechtliche Beurteilung 253

b. Außerstrafrechtliche Beurteilung 253

2. Eintritt in die Position des eigentlichen Prüfungskandidaten 255 
a. Strafrechtliche Beurteilung 255

aa. § 263 I / § 271 I StGB 255

bb. $\S 267$ I 1. und 3. Alt. StGB 255

cc. § 123 I StGB 257

b. Außerstrafrechtliche Beurteilung 257

E. Strafbarkeit des Prüflings im Falle der durch den Dritten vorgenommenen Manipulation

$\begin{array}{ll}\text { F. Heilungsproblematik } & 260\end{array}$

5. TEIL: ZUSAMMENFASSENDES ERGEBNIS UND SCHLUSSBETRACHTUNG

Anhang

Anlage

A 1. Auszug aus der Teilstudienordnung des Fachbereichs Rechtswissenschaft der Universität Trier über studienbegleitende Leistungskontrollen und Übungen während des rechtswissenschaftlichen Studiums vom 6. März 1986

A 2. Auszug aus der Promotionsordnung des Fachbereichs Rechtswissenschaft der Universität Trier vom 6. November 1979, zuletzt geändert durch Ordnung vom 7. Mai 1992

A 3. Auszug aus dem Gesetz über die Führung akademischer Grade vom 7. Juni 1939, geändert durch Art. 54 des Dritten Landesgesetzes zur Änderung strafrechtlicher Vorschriften vom 5. November 1974

A 4. Auszug aus der Verordnung zur Durchführung des Gesetzes über die Führung akademischer Grade vom 21. Juli 1939 